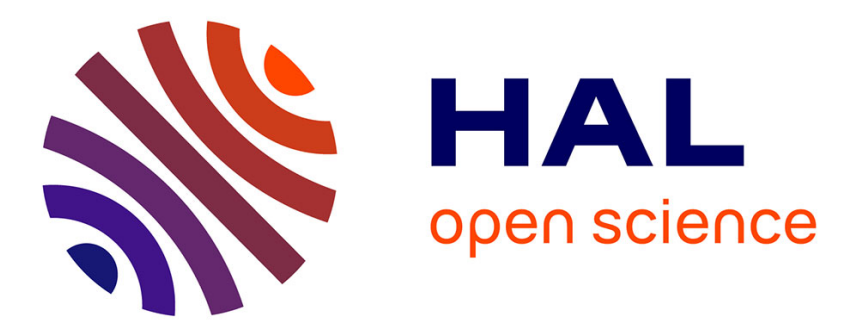

\title{
New extremal domains for the first eigenvalue of the Laplacian in flat tori
}

Pieralberto Sicbaldi

\section{To cite this version:}

Pieralberto Sicbaldi. New extremal domains for the first eigenvalue of the Laplacian in flat tori. Calculus of Variations and Partial Differential Equations, 2010, 37 (3-4), pp.329-344. 10.1007/s00526009-0264-z . hal-01266413

\section{HAL Id: hal-01266413 https://hal.science/hal-01266413}

Submitted on 2 Feb 2016

HAL is a multi-disciplinary open access archive for the deposit and dissemination of scientific research documents, whether they are published or not. The documents may come from teaching and research institutions in France or abroad, or from public or private research centers.
L'archive ouverte pluridisciplinaire HAL, est destinée au dépôt et à la diffusion de documents scientifiques de niveau recherche, publiés ou non, émanant des établissements d'enseignement et de recherche français ou étrangers, des laboratoires publics ou privés. 


\title{
NEW EXTREMAL DOMAINS FOR THE FIRST EIGENVALUE OF THE LAPLACIAN IN FLAT TORI
}

\author{
PIERALBERTO SICBALDI
}

\begin{abstract}
We prove the existence of nontrivial compact extremal domains for the first eigenvalue of the Laplacian in manifolds $\mathbb{R}^{n} \times \mathbb{R} / T \mathbb{Z}$ with flat metric, for some $T>0$. These domains are close to the cylinder-type domain $B_{1} \times \mathbb{R} / T \mathbb{Z}$, where $B_{1}$ is the unit ball in $\mathbb{R}^{n}$, they are invariant by rotation with respect to the vertical axe, and are not invariant by vertical translations. Such domains can be extended by periodicity to nontrivial and noncompact domains in Euclidean spaces whose first eigenfunction of the Laplacian with 0 Dirichlet boundary condition has also constant Neumann data at the boundary.
\end{abstract}

\section{Statement of the Result}

An open problem is to find the domains $\Omega \subseteq \mathbb{R}^{n+1}, n \geq 2$, for which the over-determined problem

$$
\left\{\begin{aligned}
\Delta_{g} u+\lambda u & =0 & & \text { in } \Omega \\
u & =0 & & \text { on } \partial \Omega \\
\stackrel{\circ}{g}(\nabla u, \nu) & =\text { constant } & & \text { on } \partial \Omega,
\end{aligned}\right.
$$

has a positive solution $u \in C^{2, \alpha}(\Omega)$. Naturally $\stackrel{\circ}{g}$ is the Euclidean metric, $\lambda$ is a positive constant (i.e. the first eigenvalue of the Laplacian), and $\nu$ is the normal unit outward vector about $\partial \Omega$. It is known (see [10]) that smooth bounded domains in Euclidean spaces for which the Laplace equation with right hand side constant, Dirichlet boundary data and constant Neumann data, are round balls. It is an open problem to study noncompact domains where this overdetermined problem is solvable.

We denote the coordinates of $\mathbb{R}^{n+1}$ as $(x, t), x \in \mathbb{R}^{n}$ and $t \in \mathbb{R}$. We want to prove the following result.

Theorem 1.1. There exist a real positive number $T_{*}<\frac{2 \pi}{\sqrt{n-1}}$, a sequence of real positive numbers $T_{j} \longrightarrow T_{*}$ and a sequence of nonzero and nonconstant functions $v_{j} \in \mathcal{C}^{2, \alpha}(\mathbb{R})$ of period $T_{j}$ converging to 0 in $\mathcal{C}^{2, \alpha}(\mathbb{R})$ such that the domains

$$
\Omega_{j}=\left\{(x, t) \in \mathbb{R}^{n+1} \quad, \quad|x|<1+v_{j}(t)\right\}
$$

have a positive solution $u_{j} \in C^{2, \alpha}\left(\Omega_{j}\right)$ to the problem (1). Moreover $\int_{0}^{T_{j}} v_{j} d t=0$. 
We remark immediately that the domain $\Omega_{j}$ can be constructed starting from the cylinder-type domain

$$
C_{1}=\left\{(x, t) \in \mathbb{R}^{n+1} \quad, \quad|x|<1\right\}
$$

(where it is also known that there exists a positive solution $u \in C^{2, \alpha}\left(C_{1}\right)$ to the problem (1)) and modifying his boundary by a function only depending on the variable $t$, periodic and nonconstant (this implies that $\Omega_{j}$ is invariant by rotations with respect to the vertical axe and is not invariant by vertical translations). Then, if $T_{j}$ is the period of the function $v_{j}$, such a domain arise to a compact domain homeomorphe to $B_{1} \times \mathbb{R} / T_{j} \mathbb{Z}$ in the manifold $\mathbb{R}^{n} \times \mathbb{R} / T_{j} \mathbb{Z}$ with flat metric, where the problem (1), adapted to this new manifold, has a solution (naturally $B_{1}$ denotes the unit ball centered at 0). From the proposition 2.1 of [9], also proved in [4] and in [3], it is clear that such a domain $\Omega_{j}$ is extremal with respect to the first eigenvalue of the Laplacian in $\mathbb{R}^{n} \times \mathbb{R} / T_{j} \mathbb{Z}$ for the fixed volume $T_{j} \operatorname{vol}\left(B_{1}\right)$, in the sens that for any volume preserving deformation $\left\{\Omega_{s}\right\}_{s \in(j-\epsilon, j+\epsilon)}$ of $\Omega_{0}$, we have

$$
\left.\frac{d \lambda_{s}}{d s}\right|_{s=0}=0
$$

where $\lambda_{s}$ is the first eigenvalue of $-\Delta_{g}$ on $\Omega_{s}$, with 0 Dirichlet boundary condition. Naturally we understood that $\left\{\Omega_{s}\right\}_{s \in(j-\epsilon, j+\epsilon)}$ is a deformation of $\Omega_{0}$, if there exists a vector field $\Xi$ such that $\Omega_{s}=\xi\left(s, \Omega_{0}\right)$ where $\xi(s, \cdot)$ is the flow associated to $\Xi$, and the deformation is said to be volume preserving if the volume of $\Omega_{s}$ does not depend on $s$.

On the other hand, given an extremal domain with respect to the first eigenvalue of the Laplacian in the manifold $\mathbb{R}^{n} \times \mathbb{R} / T \mathbb{Z}$, obtained by deformation of the boundary of the cylinder-type domain

$$
C_{1}^{T}=\left\{(x, t) \in \mathbb{R}^{n} \times \mathbb{R} / T \mathbb{Z} \quad, \quad|x|<1\right\}
$$

by a function $v(t) \in C^{2, \alpha}(\mathbb{R} / T \mathbb{Z})$ of mean 0 , then such a domain arise to a noncompact domain homeomorphe to $B_{1} \times \mathbb{R}$ defined by periodicity, where there exists a solution to the problem (1).

We can conclude that an alternative version of the theorem 1.1 is the following:

Theorem 1.2. There exists a real positive number $T_{*}<\frac{2 \pi}{\sqrt{n-1}}$, a sequence of real positive numbers $T_{j} \longrightarrow T_{*}$ and a sequence of nonzero functions $v_{j} \in \mathcal{C}^{2, \alpha}(\mathbb{R} / 2 \pi \mathbb{Z})$ of mean 0 converging to 0 in $\mathcal{C}^{2, \alpha}(\mathbb{R} / 2 \pi \mathbb{Z})$ such that the domain

$$
\Omega_{j}=\left\{(x, t) \in \mathbb{R}^{n} \times \mathbb{R} / T_{j} \mathbb{Z} \quad, \quad|x|<1+v_{j}\left(\frac{2 \pi}{T_{j}} t\right)\right\}
$$

is extremal with respect to the first eigenvalue of the Laplacian in the manifold $\mathbb{R}^{n} \times \mathbb{R} / T_{j} \mathbb{Z}$ with flat metric.

This will be our main theorem, and we will prove it.

As a final remark, we observe that this result can give an answer, with a counterexample, to the conjecture of Berestycki, Caffarelli and Nirenberg proposed in [1], p. 1110. According to this conjecture, if $\Omega$ is a smooth domain such that $\mathbb{R}^{n+1} \backslash \bar{\Omega}$ is connected and there exists a bounded 
positive solution of

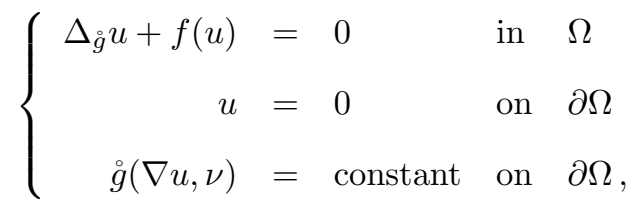

for some Lipschitz function $f$, then $\Omega$ should be either a half-space, a ball, the complement of a ball, or a circular-cylinder-type domain $\mathbb{R}^{j} \times B$, with $B$ a ball.

\section{Rephrasing the PROBLem}

We want to show that for some $T>0$ (we will see that we have to choose $T$ close to a fixed real positive number $T_{*}$ ) we can modify the boundary of $C_{1}^{T}$ in order to find an extremal domain, of volume $T \operatorname{vol}\left(B_{1}\right)$, with respect to the first eigenvalue of the Laplacian in the manifold $\mathbb{R}^{n} \times \mathbb{R} / T \mathbb{Z}$ with flat metric. Given a continuous function $f: S^{n-1} \times \mathbb{R} / T \mathbb{Z} \longmapsto(0, \infty)$ we define

$$
C_{f}^{T}:=\left\{(x, t) \in \mathbb{R}^{n} \times \mathbb{R} / T \mathbb{Z} \quad: \quad 0 \leq|x|<f(x /|x|, t)\right\} .
$$

Our aim, following the caracterization of extremal domains given in [9], is to show that there exists a $T>0$ and a nonconstant function $v: S^{n-1} \times \mathbb{R} / T \mathbb{Z} \longrightarrow \mathbb{R}$ of mean 0 such that the over-determined problem

$$
\left\{\begin{array}{rlrll}
\Delta_{\grave{g}} \phi+\lambda \phi & = & 0 & \text { in } & C_{1+v}^{T} \\
\phi & = & 0 & \text { on } & \partial C_{1+v}^{T} \\
\stackrel{g}{ }(\nabla \phi, \nu) & = & \text { constant } & \text { on } & \partial C_{1+v}^{T}
\end{array}\right.
$$

has a nontrivial positive solution, where $\nu$ is the normal vector field about $\partial C_{1+v}^{T}, \lambda$ is a positive

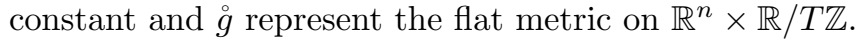

By symmetry, it is clear that the function $v$ does not depend on the variable $x \in S^{n-1}$. Then $v=v(t)$. Moreover we can also require the function $v$ to be even.

Let us denote by $\lambda_{1}$ the first eigenvalue of the euclidean Laplacian in the unit ball $B_{1}$ of $\mathbb{R}^{n}$ centered at the origin, with 0 Dirichlet boundary condition. We denote by $\tilde{\phi}_{1}$ the associated eigenfunction

$$
\left\{\begin{array}{rllll}
\Delta \tilde{\phi}_{1}+\lambda_{1} \tilde{\phi}_{1} & = & 0 & \text { in } & B_{1} \\
\tilde{\phi}_{1} & = & 0 & \text { on } & \partial B_{1}
\end{array} .\right.
$$

which is normalized to have $L^{2}\left(B_{1}\right)$-norm equal to $1 / 2 \pi$. Then $\phi_{1}(x, t)=\tilde{\phi}_{1}(x)$ solve the problem

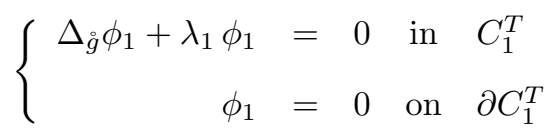


and

$$
\int_{C_{1}^{2 \pi}} \phi_{1}^{2} \mathrm{dvol}_{\stackrel{\circ}{ }}=1
$$

Because $\phi_{1}$ do not depend on $t$, sometimes we will write simply $\phi_{1}(x)$.

Let $\mathcal{C}_{\text {even, } 0}^{2, \alpha}(\mathbb{R} / 2 \pi \mathbb{Z})$ be the set of even functions on $\mathbb{R} / 2 \pi \mathbb{Z}$ of mean 0 . For all $T>0$ and all $f \in \mathcal{C}_{\text {even, } 0}^{2, \alpha}(\mathbb{R} / 2 \pi \mathbb{Z})$ we set

$$
C_{f}^{T}:=\left\{(x, t) \in \mathbb{R}^{n} \times \mathbb{R} / T \mathbb{Z} \quad: \quad 0 \leq|x|<f(2 \pi t / T)\right\} .
$$

For all function $v \in \mathcal{C}_{\text {even, } 0}^{2, \alpha}(\mathbb{R} / 2 \pi \mathbb{Z})$ whose norm is small enough, the domain $C_{1+v}^{T}$ is well defined for all $T>0$. Standard results on Dirichlet eigenvalue problem (see [5]) apply to give the existence, for all $T>0$, of a unique positive function

$$
\phi=\phi_{v, T} \in \mathcal{C}^{2, \alpha}\left(C_{1+v}^{T}\right)
$$

and a constant $\lambda=\lambda_{v, T} \in \mathbb{R}$ such that $\phi$ is a solution to the problem

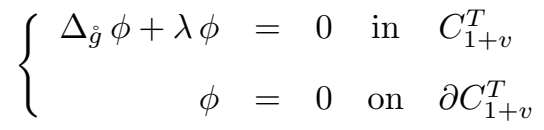

which is normalized by

$$
\int_{C_{1+v}^{2 \pi}}\left(\phi\left(x, \frac{T}{2 \pi} t\right)\right)^{2} \mathrm{dvol}_{g}=1
$$

In addition $\phi$ and $\lambda$ depend smoothly on the function $v$, and $\phi=\phi_{1}, \lambda=\lambda_{1}$ when $v \equiv 0$.

After canonical identification of $\partial C_{1+v}^{T}$ with $S^{n-1} \times \mathbb{R} / T \mathbb{Z}$, we define, the operator $N$ :

$$
N(v, T)=\left.\stackrel{\circ}{g}(\nabla \phi, \nu)\right|_{\partial C_{1+v}^{T}}-\frac{1}{\operatorname{Vol}_{g}^{g}\left(\partial C_{1+v}^{T}\right)} \int_{\partial C_{1+v}^{T}} \stackrel{\circ}{g}(\nabla \phi, \nu) \mathrm{dvol}_{\stackrel{g}{g}},
$$

where $\nu$ denotes the unit normal vector field to $\partial C_{1+v}^{T}$ and $\phi$ is the solution of (7). A priori $N(v, t)$ is a function defined over $S^{n-1} \times \mathbb{R} / T \mathbb{Z}$, but it is easy to see that it depends only on the variable $t \in \mathbb{R} / T \mathbb{Z}$ because $v$ has such a property. For the same reason it is an even function, and moreover it is clear that its mean is 0 . If now we operate a rescaling and we define

$$
F(v, T)(t)=N(v, T)\left(\frac{T}{2 \pi} t\right)
$$

Schauder's estimates imply that $F$ is well defined for $v$ in a neighborhood of 0 in $\mathcal{C}_{\text {even, } 0}^{2, \alpha}(\mathbb{R} / 2 \pi \mathbb{Z})$ and $T \in \mathbb{R}$, and takes its values in $\mathcal{C}_{\text {even, } 0}^{1, \alpha}(\mathbb{R} / 2 \pi \mathbb{Z})$. Our aim is to find $(v, T)$ such that $F(v, T)=0$. Observe that, with this condition, $\phi$ will be the solution to the problem (3).

Our next task will be to understand the structure of $L_{0}$, the operator obtained by linearizing $F$ with respect to $v$ at a general point $(0, T)$. 


\section{THE STRUCTURE OF THE LINEARIZED OPERATOR}

We already have recalled the existence of a unique positive function $\phi \in \mathcal{C}^{2, \alpha}\left(C_{1+v}^{T}\right)$ (close to $\phi_{1}$ ) and a constant $\lambda \in \mathbb{R}$ (close to $\lambda_{1}$ ), solutions to the problem (7) so we are going to construct an expansion of $\phi$ and $\lambda$ in powers of $v$ and its derivatives. This will lead to the structure of the linearized operator $L_{0}$.

Recall that $\lambda_{1}$ is the first eigenvalue of $-\Delta_{g}$ in $C_{1}^{T}$ with 0 Dirichlet boundary condition and $\phi_{1}$ is the associated eigenfunction which is normalized as in (6). Observe that in principle $\phi_{1}$ is only defined in the cylinder, however, this function being radial in the first $n$ variables and not depending on $t$, it is a solution of a second order ordinary differential equation and as such can be extended at least in a neighborhood of $\partial C_{1}^{T}$.

Let $v \in \mathcal{C}_{\text {even, }, 0}^{2, \alpha}(\mathbb{R} / 2 \pi \mathbb{Z})$. By Fourier expansion $v$ can be written as

$$
v=\sum_{k \geq 1} a_{k} \cos (k t)
$$

We start with the easy :

Lemma 3.1. Assume that $v \in \mathcal{C}_{\text {even }, 0}^{2, \alpha}(\mathbb{R} / 2 \pi \mathbb{Z})$ and write $v$ as in (9). For $T>0$ we define

$$
\phi_{0}(x, t)=\partial_{r} \phi_{1}(x) v(2 \pi t / T)
$$

where $r=|x|$. Then

$$
\Delta_{g} \phi_{0}+\lambda_{1} \phi_{0}=\sum_{k \geq 1} a_{k} \frac{1}{r^{2}} \partial_{r} \phi_{1} \cos \left(\frac{2 \pi k t}{T}\right)\left[n-1-\left(\frac{2 \pi k}{T}\right)^{2} r^{2}\right]
$$

Proof : This is a straightforward exercise. Using the fact that

$$
\Delta_{g} \partial_{r} \phi_{1}=-\lambda_{1} \partial_{r} \phi_{1}+\frac{n-1}{r^{2}} \partial_{r} \phi_{1}
$$

we find

$$
\begin{aligned}
\Delta_{g} \phi_{0} & =v \Delta_{g} \partial_{r} \phi_{1}+\partial_{r} \phi_{1} \Delta_{g} v+2 \nabla v \nabla \partial_{r} \phi_{1} \\
& =v\left(-\lambda_{1} \partial_{r} \phi_{1}+\frac{n-1}{r^{2}} \partial_{r} \phi_{1}\right)-\sum_{k \geq 1} a_{k}\left(\frac{2 \pi k}{T}\right)^{2} \partial_{r} \phi_{1} \cos \left(\frac{2 \pi k t}{T}\right) \\
& =-\lambda_{1} \phi_{0}+\sum_{k \geq 1} a_{k} \frac{1}{r^{2}} \partial_{r} \phi_{1} \cos \left(\frac{2 \pi k t}{T}\right)\left[n-1-\left(\frac{2 \pi k}{T}\right)^{2} r^{2}\right]
\end{aligned}
$$

This completes the proof of the result. 
For all $v \in \mathcal{C}_{\text {even }, 0}^{2, \alpha}(\mathbb{R} / 2 \pi \mathbb{Z})$ and all $T>0$ let $\psi$ be the (unique) solution (periodic with respect to the variable $t$ ) of

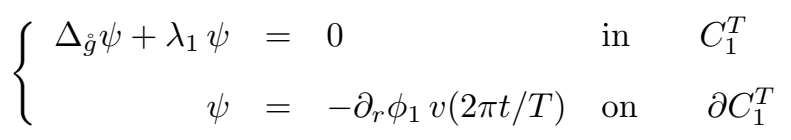

which is $L^{2}\left(C_{1}^{T}\right)$-orthogonal to $\phi_{1}$. We define

$$
\tilde{H}_{T}(v):=\left.\left(\partial_{r} \psi+\partial_{r}^{2} \phi_{1} v(2 \pi t / T)\right)\right|_{\partial C_{1}^{T}}
$$

By symmetry it is clear that $\tilde{H}_{T}(v)$ is a function only depending on $t$, then changing the variable we can define

$$
H_{T}(v)(t):=\tilde{H}_{T}(v)\left(\frac{T}{2 \pi} t\right)
$$

Let $V_{k}$ be the space spanned by the function $\cos (k t)$. We will need the following result :

Proposition 3.2. The operator

$$
H_{T}: \mathcal{C}_{\text {even }, 0}^{2, \alpha}(\mathbb{R} / 2 \pi \mathbb{Z}) \longrightarrow \mathcal{C}_{\text {even }, 0}^{1, \alpha}(\mathbb{R} / 2 \pi \mathbb{Z})
$$

is a self adjoint, first order elliptic operator that preserves the eigenspaces $V_{k}$ for all $k$ and all $T>0$. There exists a positive real number $T_{*}<\frac{2 \pi}{\sqrt{n-1}}$ such that the kernel of $H_{T_{*}}$ is given by $V_{k_{1}} \oplus \cdots \oplus V_{k_{l}}$, with $1=k_{1}<k_{2}<\cdots<k_{l}$. Moreover the eigenvalue associated to the eigenspace $V_{1}$, considered as a function on $T$, changes the sign at $T_{*}$, and the eigenvalues associated to the other eigenspaces $V_{k_{2}}, \ldots, V_{k_{l}}$, always considered as functions on $T$, do not change the sign at $T_{*}$. There exists a constant $c>0$ such that

$$
\|w\|_{\mathcal{C}^{2, \alpha}(\mathbb{R} / 2 \pi \mathbb{Z})} \leq c\left\|H_{T_{*}}(w)\right\|_{\mathcal{C}^{1, \alpha}(\mathbb{R} / 2 \pi \mathbb{Z})},
$$

provided $w$ is $L^{2}(\mathbb{R} / 2 \pi \mathbb{Z})$-orthogonal to $V_{0} \oplus V_{k_{1}} \oplus \cdots \oplus V_{k_{l}}$, where $V_{0}$ is the space of constant functions.

Proof : The fact that $H_{T}$ is a first order elliptic operator is standard since it is the sum of the Dirichlet-to-Neumann operator for $\Delta_{g}+\lambda_{1}$ and a constant times the identity. In particular, elliptic estimates yield

$$
\left\|H_{T}(w)\right\|_{\mathcal{C}^{1, \alpha}(\mathbb{R} / 2 \pi \mathbb{Z})} \leq c\|w\|_{\mathcal{C}^{2, \alpha}(\mathbb{R} / 2 \pi \mathbb{Z})}
$$

The fact that the operator $H_{T}$ is (formally) self-adjoint is easy. Let $\psi_{1}$ (resp. $\psi_{2}$ ) the solution of (11) corresponding to the function $w_{1}$ (resp. $\left.w_{2}\right)$. Let $\tilde{\psi}_{i}(x, t)=\psi_{i}(x, T t / 2 \pi)$. We compute

$$
\begin{aligned}
\partial_{r} \phi_{1}(1) \int_{0}^{2 \pi}\left(H_{T}\left(w_{1}\right) w_{2}-w_{1} H_{T}\left(w_{2}\right)\right) d t & =\partial_{r} \phi_{1}(1) \int_{0}^{2 \pi}\left(\partial_{r} \tilde{\psi}_{1} w_{2}-\partial_{r} \tilde{\psi}_{2} w_{1}\right) d t \\
& =\int_{0}^{2 \pi}\left(\tilde{\psi}_{1} \partial_{r} \tilde{\psi}_{2}-\tilde{\psi}_{2} \partial_{r} \tilde{\psi}_{1}\right) d t \\
& =\frac{1}{\operatorname{Vol}_{\grave{g}}\left(S^{n-1}\right)} \int_{C_{1}^{2 \pi}}\left(\tilde{\psi}_{1} \Delta_{\dot{g}} \tilde{\psi}_{2}-\tilde{\psi}_{2} \Delta_{\grave{g}} \tilde{\psi}_{1}\right) \mathrm{dvol}_{\grave{g}} \\
& =0
\end{aligned}
$$


To prove the other statements, we define for all $v \in \mathcal{C}_{\text {even }, 0}^{2, \alpha}(\mathbb{R} / 2 \pi \mathbb{Z})$ written as in $(9)$, $\Psi$ to be the continuous solution of

$$
\left\{\begin{array}{rl}
\Delta_{\grave{g}} \Psi+\lambda_{1} \Psi & =\sum_{k \geq 1} a_{k} \frac{1}{r^{2}} \partial_{r} \phi_{1} \cos \left(\frac{2 \pi k t}{T}\right)\left[n-1-\left(\frac{2 \pi k}{T}\right)^{2} r^{2}\right] \text { in } \begin{array}{c}
C_{1}^{T} \\
\Psi
\end{array}=0 \quad \text { on } \partial C_{1}^{T} .
\end{array} .\right.
$$

Observe that $\partial_{r} \phi_{1}$ vanishes at first order at $r=0$ and hence the right hand side is bounded by a constant times $r^{-1}$ near the origin. Standard elliptic estimates then imply that the solution $\Psi$ is at least continuous near the origin (the right side of (14) belongs to the space $L^{p}\left(C_{1}^{T}\right)$ for each $p<n$, then the solution $\Psi$ belongs to the Sobolev space $W^{2, p}\left(C_{1}^{T}\right)$ for each $p<n$, and by the Sobolev embedding theorem for a compact domain $\Omega$ we have $W^{2, p}(\Omega) \subseteq C^{0, \alpha}(\Omega)$ for $\left.p \geq \frac{n}{2-\alpha}\right)$. A straightforward computation using the result of Lemma 3.1 and writing $\Psi(x, t)=$ $\psi(x, t)+\partial_{r} \phi_{1}(x) v(2 \pi t / T)$, shows that

$$
\tilde{H}_{T}(v):=\left.\partial_{r} \Psi\right|_{\partial C_{1}^{T}}
$$

With this alternative definition, it should be clear that $H_{T}$ preserves the eigenspaces $V_{k}$ and in particular, $H_{T}$ maps into the space of functions whose mean is 0 .

Then

$$
\tilde{H}_{T}(v)=\sum_{k \geq 1} \sigma_{k}(T) a_{k} \cos \left(\frac{2 \pi k t}{T}\right)
$$

where $\sigma_{k}(T)$ are the eigenvalues of $H_{T}$ with respect to the eigenfunctions $\cos (k t)$ and are given by

$$
\sigma_{k}(T)=\partial_{r} b_{k}(1)
$$

where $b_{k}$ is the continuous solution on $[0,1]$ of

$$
\left(\partial_{r}^{2}+\frac{n-1}{r} \partial_{r}+\lambda_{1}\right) b_{k}-\left(\frac{2 \pi k}{T}\right)^{2} b_{k}=\frac{1}{r^{2}}\left[n-1-\left(\frac{2 \pi k}{T}\right)^{2} r^{2}\right] \partial_{r} \phi_{1},
$$

with $b_{k}(1)=0$. From (12), (16) and (11) we deduce that

$$
\psi=\sum_{k \geq 1} c_{k}(r) a_{k} \cos \left(\frac{2 \pi k t}{T}\right)
$$

where $c_{k}$ is the continuous solution on $[0,1]$ of

$$
\left(\partial_{r}^{2}+\frac{n-1}{r} \partial_{r}+\lambda_{1}\right) c_{k}-\left(\frac{2 \pi k}{T}\right)^{2} c_{k}=0
$$

with $c_{k}(1)=-\partial_{r} \phi_{1}(1)$. Then an alternative characterization of the eigenvalue $\sigma_{k}(T)$ is

$$
\sigma_{k}(T)=\partial_{r} c_{k}(1)+\partial_{r}^{2} \phi_{1}(1)
$$

We want to show that there exists $T_{*}>0$ such that the kernel of $H_{T_{*}}$ is finite-dimensional and contains $V_{1}$, the space of functions of the form $a_{1} \cos (t)$. For this aim we have to find a $T_{*}>0$ such that $\sigma_{1}\left(T_{*}\right)=0$ and $\sigma_{k}\left(T_{*}\right) \neq 0$ for almost $k>1$. 
To simplify the notation, we set $\sigma_{1}=\sigma$. We need the following Lemma :

Lemma 3.3. The function $\sigma(T)$ is analytic on $(0,+\infty)$ and has the following properties:

- $\lim _{T \rightarrow 0^{+}} \sigma(T)=+\infty$

- $\sigma(T)<0$ for $T \geq \frac{2 \pi}{\sqrt{n-1}}$

In particular $\sigma$ has at least a zero where it changes the sign, and the set of the zeros of $\sigma$ is a discrete finite set.

Proof : The fact that $\sigma$ is analytic comes from the following remark : if $F$ is an invertible operator and $I$ is the identity, then for $T>0$ and any continuous function $v$ the solution $u$ of

$$
\left(F-\frac{1}{T^{2}} \rho I\right) u=v
$$

is analytic on $T$ for each constant $\rho$; this comes from the equality

$$
(I-s F)^{-1}=\sum_{n \geq 0} s^{n} F^{n}
$$

for each $s \in \mathbb{R}$. Then to prove that $c_{1}$ is analytic on $T$ it suffices to take

$$
F=\left(\partial_{r}^{2}+\frac{n-1}{r} \partial_{r}+\lambda_{1}\right) \quad v=0 \quad \rho=(2 \pi)^{2}
$$

We conclude that $\partial_{r} c_{1}(1)$ is analytic with respect to $T$, and from (20) follows the analyticity of $\sigma$.

We study now the behaviour of $\sigma$ when $T \rightarrow 0^{+}$. For $T$ small enough, it is well defined the quantity

$$
\xi=\sqrt{\frac{4 \pi^{2}}{T^{2}}-\lambda_{1}}
$$

Let $\tilde{c}(s)=c_{1}\left(\frac{s}{\xi}\right)$ for $s \in[0, \xi]$. We remark that

$$
\lim _{T \rightarrow 0^{+}} \partial_{r} c_{1}(1)=\lim _{\xi \rightarrow+\infty} \partial_{s} \tilde{c}(\xi)
$$

Then we are interested in the behaviour of $\partial_{s} \tilde{c}(\xi)$ at $+\infty$. From (19) (considered for $k=1$ ) we obtain that $\tilde{c}$ is a continuous solution of the differential equation

$$
\left(\partial_{s}^{2}+\frac{n-1}{s} \partial_{s}-1\right) \tilde{c}=0
$$

in the interval $[0, \xi]$ with $\tilde{c}(\xi)=-\partial_{r} \phi_{1}(1)$. The previuos equation can be transformed into a well known Bessel's differential equation by the substitution

$$
\tilde{c}=s^{\frac{2-n}{2}} \hat{c}
$$


It follows that $\hat{c}$ satisfies

$$
\left[\partial_{s}^{2}+\frac{1}{s} \partial_{s}-\left(1+\frac{\left(\frac{2-n}{2}\right)^{2}}{s^{2}}\right)\right] \tilde{c}=0
$$

that is the modified Bessel's differential equation of order $\frac{2-n}{2}$ (for an introduction to Bessel's equations see for example [2] or [6]), and its general solution is given by

$$
\hat{c}(s)=A I_{\frac{2-n}{2}}(s)+B K_{\frac{2-n}{2}}(s)
$$

for some constants $A, B \in \mathbb{R}$, where $I_{m}(s)$ and $K_{m}(s)$ (for $m \in \mathbb{R}$ ) are the well known modified Bessel functions given by

$$
\begin{aligned}
I_{m}(s) & =\sum_{k=0}^{\infty} \frac{1}{k ! \Gamma(m+k+1)}\left(\frac{s}{2}\right)^{m+2 k}, \\
K_{m}(s) & =\lim _{p \rightarrow m} \frac{\pi}{2}\left[\frac{I_{-p}(s)-I_{p}(s)}{\sin (p s)}\right]
\end{aligned},
$$

where $\Gamma$ is the Gamma function

$$
\Gamma(s)=\int_{0}^{\infty} e^{-t} t^{s-1} d t
$$

$I_{m}$ and $K_{m}$ are independent solutions of the modified Bessel's differential equation of order $m$

$$
\partial_{s}^{2} f+\frac{1}{s} \partial_{s} f-\left(1+\frac{m^{2}}{s^{2}}\right) f=0
$$

It is well known that the behaviour of $I_{m}$ at $\infty$ is

$$
\lim _{s \rightarrow+\infty} \frac{I_{m}(s)}{\frac{1}{\sqrt{2 \pi s}} e^{s}}=1,
$$

while that of $K_{m}$ is

$$
\lim _{s \rightarrow+\infty} \frac{K_{m}(s)}{\sqrt{\frac{\pi}{2 s}} e^{-s}}=1 .
$$

From (22) and (24) we have that

$$
\tilde{c}(s)=s^{\frac{2-n}{2}}\left(A I_{\frac{2-n}{2}}(s)+B K_{\frac{2-n}{2}}(s)\right)
$$

The behaviour of $\tilde{c}$ at $\infty$ can't be that of the function $s^{\frac{2-n}{2}} K_{\frac{2-n}{2}}(s)$ because of the continuity of $\tilde{c}$ at 0 . In fact, if it was like that then we could integrate over $[0,+\infty]$ the function $\tilde{c}$ with its derivatives, and from (21), multiplying by $\tilde{c} s^{n-1}$ and integrating by parts, we would obtain

$$
\int_{0}^{+\infty}\left(\left(\partial_{s} \tilde{c}\right)^{2}+\tilde{c}^{2}\right) s^{n-1} d s=0
$$


that would imply $\tilde{c}=0$. Hence, by (25) and (26), the behaviour of $\tilde{c}$ when $s \rightarrow+\infty$ is that of the function $s^{\frac{2-n}{2}} I_{\frac{2-n}{2}}(s)$, i.e. there exixts a constant $\tilde{A} \neq 0$ such that

$$
\lim _{s \rightarrow+\infty} \frac{\tilde{c}(s)}{\tilde{A} s^{\frac{1-n}{2}} e^{s}}=1 .
$$

It follows that $\partial_{s} \tilde{c}(\xi) \rightarrow+\infty$ when $\xi \rightarrow+\infty$, and then, coming back to the definition of $\xi$, we conclude that

$$
\lim _{T \rightarrow 0^{+}} \sigma(T)=+\infty
$$

Now we will show that for $T$ big enough the function $\sigma$ is negative. Let $A_{T}(r)$ be the solution of the following differential equation

$$
\partial_{r}^{2} A_{T}+\frac{n-1}{r} \partial_{r} A_{T}+\left[\lambda_{1}-\left(\frac{2 \pi}{T}\right)^{2}\right] A_{T}=\frac{1}{r^{2}} \partial_{r} \phi_{1},
$$

and $B_{T}(r)$ be the solution of this other differential equation

$$
\partial_{r}^{2} B_{T}+\frac{n-1}{r} \partial_{r} B_{T}+\left[\lambda_{1}-\left(\frac{2 \pi}{T}\right)^{2}\right] B_{T}=\partial_{r} \phi_{1},
$$

with $A_{T}(1)=B_{T}(1)=0$. It is easy to see that

$$
b_{1}=(n-1) A_{T}-\left(\frac{2 \pi}{T}\right)^{2} B_{T}
$$

We have

$$
\sigma(T)=(n-1) \partial_{r} A_{T}(1)-\left(\frac{2 \pi}{T}\right)^{2} \partial_{r} B_{T}(1)
$$

We claim that $A_{T} \geq 0, B_{T} \geq 0$ and $A_{T}-B_{T} \geq 0$. Moreover $\partial_{r} A_{T}(1)<0, \partial_{r} B_{T}(1)<0$ and $\partial_{r} A_{T}(1)<\partial_{r} B_{T}(1)$. This follows from the maximum principle.

Proof of the claim : By definition of $\lambda_{1}$, the operator $\Delta_{g}+\lambda_{1}$ is non-positive, in the sense that

$$
-\int_{B_{1}} u\left(\Delta_{\grave{g}}+\lambda_{1}\right) u \mathrm{dvol}_{\grave{g}}=\int_{B_{1}}\left(|\nabla u|_{\grave{g}}^{2}-\lambda_{1} u^{2}\right) \mathrm{dvol}_{\grave{g}} \geq 0 .
$$

Specializing this inequality to functions $u(x, t)=u(|x|)$, we get

$$
\int_{0}^{1}\left(\left(\partial_{r} u\right)^{2}-\lambda_{1} u^{2}\right) r^{n-1} \mathrm{dr} \geq 0
$$

where $r=|x|$, provided $u \in H_{0}^{1}\left(B_{1}\right)$. Assume now that $A_{T} \leq 0$ in $\left[r_{1}, r_{2}\right]$ with $A_{T}\left(r_{i}\right)=0$, then multiplying (27) by $A_{T} r^{n-1}$ and integrating the result by parts between $r_{1}$ and $r_{2}$, we get

$$
\int_{r_{1}}^{r_{2}}\left(\left(\partial_{r} A_{T}\right)^{2}-\lambda_{1} A_{T}^{2}\right) r^{n-1} \mathrm{dr}+\left(\frac{2 \pi}{T}\right)^{2} \int_{r_{1}}^{r_{2}} A_{T}^{2} r^{n-1} \mathrm{dr} \leq 0
$$

because $\partial_{r} \phi_{1} \leq 0$. Hence, by (30), necessarily $A_{T} \equiv 0$ on $\left[r_{1}, r_{2}\right]$. This proves that $A_{T} \geq 0$ on $\left[r_{1}, r_{2}\right]$ and by the maximum priciple $A_{T} \geq 0$ on $[0,1]$. It follows from this fact that $\partial_{r} A_{T}(1) \leq 0$, 
because $A_{T}(1)=0$. If it was $\partial_{r} A_{T}(1)=0$ then necessarily we would have $\partial_{r}^{2} A_{T}(1) \geq 0$ but evaluation of (27) at $r=1$ implies that

$$
0=(n-1) \partial_{r} A_{T}(1)=\partial_{r} \phi_{1}(1)-\partial_{r}^{2} A_{T}(1) \leq \partial_{r} \phi_{1}(1)<0
$$

which immediately leads to a contradiction. Hence, $\partial_{r} A_{T}(1)<0$. The same reasoning applies starting from (28) to show that $B_{T} \geq 0$ and $\partial_{r} B_{T}(1)<0$, and starting from the difference between (27) and (28) to show that $A_{T}-B_{T} \geq 0$ with $\partial_{r}\left(A_{T}-B_{T}\right)<0$. This completes the proof of the claim.

Let now

$$
T \geq \frac{2 \pi}{\sqrt{n-1}}
$$

From the previous claim we have

$$
-\left(\frac{2 \pi}{T}\right)^{2} \partial_{r} B_{T} \leq-(n-1) \partial_{r} B_{T}<-(n-1) \partial_{r} A_{T}
$$

that means

$$
\sigma(T)<0
$$

This completes the proof of the Lemma.

Let $\left\{0_{1}, 0_{2}, \ldots, 0_{p}\right\}$ the finite set of the zeros of $\sigma$, and let $T_{*}$ the smallest zero such that $\sigma$ changes the sign at $T_{*}$, say that $T_{*}=0_{q}$. It is clear then $V_{1}$ is in the Kernel of $H_{T_{*}}$. To prove that the kernel of $H_{T_{*}}$ is finite-dimensional we have to show that if $T=T_{*}$ then $\partial_{r} b_{k}(1) \neq 0$ for almost $k>1$. For this we set

$$
\frac{k}{T}=\frac{1}{\tau}
$$

for $T>0$ and from (18) we obtain that

$$
\sigma_{k}(T)=\sigma(\tau)
$$

This implies that $\sigma_{k}$ is analytic on $T$ and the set of the zeros of $\sigma_{k}$ is $\left\{k 0_{1}, k 0_{2}, \ldots, k 0_{p}\right\}$. It is clear that if $k$ is big enough, say $k>k_{l}$, then $T_{*} \notin\left\{k 0_{1}, k 0_{2}, \ldots, k 0_{p}\right\}$, and this means that $V_{k}$ is not a kernel of $H_{T_{*}}$ for $k>k_{l}$. This implies that the kernel of $H_{T_{*}}$ is of the form $V_{k_{1}} \oplus \cdots \oplus V_{k_{l}}$ with $1=k_{1}<\cdots<k_{l}$. Moreover if $V_{k_{i}} \in \operatorname{Ker}\left(H_{T_{*}}\right)$ and $k_{i} \neq 1$ then the function $\sigma_{k_{i}}(T)$ does not change the sign at $T_{*}$ because $\sigma_{k_{i}}\left(T_{*}\right)=\sigma\left(T_{*} / k_{i}\right)$ and $T_{*} / k_{i}<T_{*}$. This completes the proof of the proposition 3.2 .

The main result of this section is the following :

Proposition 3.4. The operator $L_{0}$ is equal to $H_{T}$.

Proof : By definition, the operator $L_{0}$ is the linear operator obtained by linearizing $F$ with respect to $v$ at $(0, T)$. In other words, we have

$$
L_{0}(w)=\lim _{s \rightarrow 0} \frac{F(s w, T)-F(0, T)}{s} .
$$


For $y \in \mathbb{R}^{n}$ and $t \in \mathbb{R}$ we consider the parameterization of $C_{1+v}^{T}$ given by

$$
Y(y, t):=\left((1+s \chi(y) w) y, \frac{T t}{2 \pi}\right)
$$

where $\chi$ is a cutoff function identically equal to 0 when $|y| \leq 1 / 2$ and identically equal to 1 when $|y| \geq 3 / 4$. We set

$$
\hat{g}:=Y^{*} \stackrel{\circ}{g}
$$

so that $\hat{\phi}=Y^{*} \phi$ and $\hat{\lambda}=\lambda$ are solutions (smoothly depending on the real parameter $s$ ) of

$$
\left\{\begin{array}{rlll}
\Delta_{\hat{g}} \hat{\phi}+\hat{\lambda} \hat{\phi} & =0 & \text { in } & C_{1}^{2 \pi} \\
\hat{\phi} & =0 & \text { on } & \partial C_{1}^{2 \pi}
\end{array}\right.
$$

with

$$
\int_{C_{1}^{2 \pi}} \hat{\phi}^{2} \operatorname{dvol}_{\hat{g}}=1
$$

We remark that $\hat{\phi}_{1}:=Y^{*} \phi_{1}$ is a solution of

$$
\Delta_{\hat{g}} \hat{\phi}_{1}+\lambda_{1} \hat{\phi}_{1}=0
$$

since $\hat{g}=Y^{*} \stackrel{\circ}{g}$. Moreover

$$
\hat{\phi}_{1}(y, t)=\phi_{1}\left((1+s w) y, \frac{T t}{2 \pi}\right),
$$

on $\partial C_{1}^{2 \pi}$. Writing $\hat{\phi}=\hat{\phi}_{1}+\hat{\psi}$ and $\hat{\lambda}=\lambda_{1}+\mu$, we find that

$$
\left\{\begin{array}{rlrl}
\Delta_{\hat{g}} \hat{\psi}+\left(\lambda_{1}+\mu\right) \hat{\psi}+\mu \hat{\phi}_{1} & =0 & \text { in } C_{1}^{2 \pi} \\
\hat{\psi} & =-\hat{\phi}_{1} \text { on } \partial C_{1}^{2 \pi}
\end{array}\right.
$$

with

$$
\int_{C_{1}^{2 \pi}}\left(2 \hat{\phi}_{1} \hat{\psi}+\hat{\psi}^{2}\right) \operatorname{dvol}_{\hat{g}}=\int_{C_{1}^{2 \pi}} \phi_{1}^{2} \operatorname{dvol}_{\grave{g}}-\int_{C_{1+s w}^{2 \pi}} \phi_{1}^{2} \operatorname{dvol}_{\grave{g}}
$$

Obviously $\hat{\psi}$ and $\mu$ are smooth functions of $s$. When $s=0$, we have $\phi=\phi_{1}$ and $\lambda=\lambda_{1}$. Therefore, $\hat{\psi}$ and $\mu$ vanish and $\hat{\phi}_{1}=\phi_{1}$, when $s=0$. Moreover $\hat{g}=\stackrel{\circ}{g}$ when $s=0$. We set

$$
\dot{\psi}=\left.\partial_{s} \hat{\psi}\right|_{s=0}, \quad \text { and } \quad \dot{\mu}=\left.\partial_{s} \mu\right|_{s=0},
$$

Differentiating (32) with respect to $s$ and evaluating the result at $s=0$, we obtain

$$
\left\{\begin{aligned}
\Delta_{\dot{g}} \dot{\psi}+\lambda_{1} \dot{\psi}+\dot{\mu} \phi_{1} & =0 & & \text { in } C_{1}^{2 \pi} \\
\dot{\psi} & =-\partial_{r} \phi_{1} w & \text { on } & \partial C_{1}^{2 \pi}
\end{aligned}\right.
$$

because from (31), differentiation with respect to $s$ at $s=0$ yields $\left.\partial_{s} \hat{\phi}_{1}\right|_{s=0}=\partial_{r} \phi_{1} w$. 
Differentiating (33) with respect to $s$ and evaluating the result at $s=0$, we obtain

$$
\int_{C_{1}^{2 \pi}} \phi_{1} \dot{\psi} \operatorname{dvol}_{g}=0
$$

Indeed, the derivative of the right hand side of (33) with respect to $s$ vanishes when $s=0$ since $\phi_{1}$ vanishes identically on $\partial C_{1}^{2 \pi}$.

If we multiply the first equation of (34) by $\phi_{1}$ and we integrate it over $C_{1}^{2 \pi}$, using the boundary condition and the fact that the average of $w$ is 0 we conclude that $\dot{\mu}=0$. And hence $\dot{\psi}(2 \pi t / T)$ is precisely the solution of (11). To summarize, we have proven that

$$
\hat{\phi}(x, t)=\hat{\phi}_{1}(x, t)+s \psi(x, T t / 2 \pi)+\mathcal{O}\left(s^{2}\right)
$$

where $\psi$ is the solution of (11). In particular, in $C_{1}^{2 \pi} \backslash C_{3 / 4}^{2 \pi}$, we have

$$
\begin{aligned}
\hat{\phi}(y, t) & =\phi_{1}((1+s w) y, T t / 2 \pi)+s \psi(y, T t / 2 \pi)+\mathcal{O}\left(s^{2}\right) \\
& =\phi_{1}(y, T t / 2 \pi)+s\left(w r \partial_{r} \phi_{1}+\psi(y, T t / 2 \pi)\right)+\mathcal{O}\left(s^{2}\right)
\end{aligned}
$$

where we have set $r:=|y|$.

To complete the proof of the result, it suffices to compute the normal derivative of the function $\hat{\phi}$ when the normal is computed with respect to the metric $\hat{g}$. We use cylindrical coordinates $(y, t)=(r z, t)$ where $r>0$ and $z \in S^{n-1}$. Then the metric $\hat{g}$ can be expanded in $C_{1}^{2 \pi} \backslash C_{3 / 4}^{2 \pi}$ as

$$
\hat{g}=(1+s w)^{2} d r^{2}+s r w^{\prime}(1+s w) d r d t+\left((T / 2 \pi)^{2}+s^{2} r^{2}\left(w^{\prime}\right)^{2}\right) d t^{2}+r^{2}(1+s w)^{2} h
$$

where $h$ is the metric on $S^{n-1}$ induced by the Euclidean metric. It follows from this expression that the unit normal vector field to $\partial C_{1}^{2 \pi}$ for the metric $\hat{g}$ is given by

$$
\hat{\nu}=\left((1+s w)^{-1}+\mathcal{O}\left(s^{2}\right)\right) \partial_{r}+\mathcal{O}(s) \partial_{t}
$$

Using this, we conclude that

$$
\hat{g}\left(\nabla \hat{\phi}_{1}, \hat{\nu}\right)=\partial_{r} \phi_{1}+s\left(w \partial_{r}^{2} \phi_{1}+\partial_{r} \psi(y, T t / 2 \pi)\right)+\mathcal{O}\left(s^{2}\right)
$$

on $\partial C_{1}^{2 \pi}$. The result then follows at once from the fact that $\partial_{r} \phi_{1}$ is constant while the term $w \partial_{r}^{2} \phi_{1}+\partial_{r} \psi(y, T t / 2 \pi)$ has mean 0 on the boundary $\partial C_{1}^{2 \pi}$. This completes the proof of the proposition.

\section{A Lyapunov-Schmidt ARgument}

Our aim is to prove that for some $T \in(0,+\infty)$ there exists a nonzero (and obviously nonconstant) function $v$ that solves the equation

$$
F(v, T)=0 .
$$

Unfortunately we will not be able to solve this equation at once. Instead we can split the image of the operator $F$ into two spaces, one infinite-dimensional and one finite-dimensional, and solve the equation over the infinite-dimensional space. 
It follows from the previous paragraph that the kernel of the operator $H_{T_{*}}$ is finite-dimensional and given by $V_{k_{1}} \oplus \cdots \oplus V_{k_{l}}$. Based on this fact we will be interested in the method of LyapunovSchmidt, that is a procedure to reduce the dimension of the space in which we try to solve our equation $F(v, T)=0$ near a singular point from infinite to finite dimension. The idea is to split the space into two subspaces and to project the equation into each one of them. One of the two equations obtained can be solved by the implicit function theorem.

Let $Q$ be the projection operator onto the image of $H_{T_{*}}$ and $Q \circ F$ the composition of operators $F$ and $Q$. Let $v=v^{\|}+v^{\perp}$ with $v^{\perp} \in\left(\operatorname{Ker} H_{T_{*}}\right)^{\perp}$ for a generical function in $\mathcal{C}_{\text {even, } 0}^{2, \alpha}(\mathbb{R} / 2 \pi \mathbb{Z})$. The next result follows from the implicit function theorem :

Proposition 4.1. For all $v^{\|} \in\left(\operatorname{Ker} H_{T_{*}}\right)$ which norm is small enough and all $T$ sufficiently close to $T_{*}$ there exists a unique function $v^{\perp}=v^{\perp}\left(v^{\|}, T\right)$ defined in a neighborhood of $\left(0, T_{*}\right)$ such that

$$
Q \circ F\left(v^{\|}+v^{\perp}, T\right)=0 .
$$

Proof : We can define the operator

$$
J\left(v^{\|}, v^{\perp}, T\right)=Q \circ F\left(v^{\|}+v^{\perp}, T\right)
$$

The operator $J$ maps from $\operatorname{Ker} H_{T_{*}} \times\left(\operatorname{Ker} H_{T_{*}}\right)^{\perp} \times(0,+\infty)$ into the image of $H_{T_{*}}$. By the proposition 3.2 the implicit function theorem applies to get the existence of a unique function $v^{\perp}\left(v^{\|}, T\right) \in\left(\operatorname{Ker} H_{T_{*}}\right)^{\perp}$ smoothly depending on $v^{\|}$and $T$ in a neighborhood of $\left(0, T_{*}\right)$ such that

$$
J\left(v^{\|}, v^{\perp}\left(v^{\|}, T\right), T\right)=0 .
$$

\section{A Bifurcation ARgument}

We are now able to prove our main theorem 1.2. We will use a bifurcation argument. For the sake of completeness we recall the concept of bifurcation and bifurcation point (see [8] and [11] for details). Let $f$ be an operator on $\mathbb{B}_{1} \times \Lambda$ into $\mathbb{B}_{2}$, where $\mathbb{B}_{1}$ and $\mathbb{B}_{2}$ are Banach spaces (or subspaces) and $\Lambda$ is an interval of $\mathbb{R}$. Thus suppose that $\Gamma=(x(s), s)$ is a curve of solutions of the equation $f(x, s)=0$. Let $\left(x_{0}, s_{0}\right)=\left(x\left(s_{0}\right), s_{0}\right)$ be an interior point on this curve with the property that every neighborhood of $\left(x_{0}, s_{0}\right)$ in $\mathbb{B}_{1} \times \Lambda$ contains solutions of the equation $f(x, s)=0$ which are not in $\Gamma$. Then $\left(x_{0}, s_{0}\right)$ is called a bifurcation point with respect to $\Gamma$ and we say that in that point there is a bifurcation of the solution of $f(x, s)=0$.

In relation to our problem we will show that $\left(0, T_{*}\right)$ is a bifurcation point with respect to the curve $\Gamma=(0, T)$ for the solution of the equation $F(v, T)=0$. Here $\Lambda=(0,+\infty)$, $\mathbb{B}_{1}=\mathcal{C}_{\text {even }, 0}^{2, \alpha}(\mathbb{R} / 2 \pi \mathbb{Z})$ and $\mathbb{B}_{2}=\mathcal{C}_{\text {even }, 0}^{1, \alpha}(\mathbb{R} / 2 \pi \mathbb{Z})$. We remark that this is equivalent to the existence of a sequence of real positive numbers $T_{j} \longrightarrow T_{*}$ and a sequence of functions $v_{j} \in \mathcal{C}_{\text {even, } 0}^{2, \alpha}(\mathbb{R} / 2 \pi \mathbb{Z})$ converging to 0 in $\mathcal{C}^{2, \alpha}(\mathbb{R} / 2 \pi \mathbb{Z})$ such that the points $\left(v_{j}, T_{j}\right)$ are solutions of the equation $F(v, T)=0$. And such a result is exactly our main theorem 1.2 (moreover the functions $v_{j}$ are even). 
We have proven that for each $\left(v^{\|}, T\right)$ in a neighborhood of $\left(0, T_{*}\right)$ there exists a function $v^{\perp} \in \operatorname{Ker}\left(H_{T_{*}}\right)^{\perp}$ smoothly depending on $v^{\|}$and $T$ such that

$$
Q \circ F\left(v^{\|}+v^{\perp}\left(v^{\|}, T\right), T\right)=0 .
$$

Let $I$ be the identity operator. We remark that if we find $\left(v^{\|}, T\right)$ such that

$$
(I-Q) \circ F\left(v^{\|}+v^{\perp}\left(v^{\|}, T\right), T\right)=0 .
$$

then it will be verified the equation

$$
F\left(v^{\|}+v^{\perp}\left(v^{\|}, T\right), T\right)=0
$$

Let us define

$$
G\left(v^{\|}, T\right)=(I-Q) \circ F\left(v^{\|}+v^{\perp}\left(v^{\|}, T\right), T\right)
$$

We will prove that $\left(0, T_{*}\right)$ is a bifurcation point with respect to the curve

$$
\left\{(0, T) \quad, \quad T \text { in a neighborhood of } T_{*}\right\}
$$

for the finite dimensional operator $G$. It will follow the existence of a sequence of real positive numbers $T_{j} \longrightarrow T_{*}$ and a sequence of functions $v_{j}^{\|}$converging to 0 such that the points $\left(v_{j}^{\|}, T_{j}\right)$ are solutions of the equation $G\left(v^{\|}, T\right)=0$. Then the sequence $\left(v_{j}, T_{j}\right)$ with $v_{j}$ defined by

$$
v_{j}=v_{j}^{\|}+v^{\perp}\left(v_{j}^{\|}, T\right)
$$

will satisfy the statement of the main theorem 1.2.

We recall the concept of odd crossing number following the approach of [8]. Let we come back to the operator $f$, with finite-dimensional Banach spaces $\mathbb{B}_{1}$ and $\mathbb{B}_{2}$. A necessary condition for bifurcation is that 0 is an isolated eigenvalue of finite algebraic multiplicity, say $l$, of the operator obtained by linearizing $f$ with respect to $x$ at $\left(0, s_{0}\right)$, which can be denoted by $D_{x} f\left(0, s_{0}\right)$. It is crucial to know how the eigenvalue 0 perturbs for $D_{x} f\left(0, s_{0}\right)$ when $s$ varies in a neighborhood of $s_{0}$. It is possible to show (see [7]) that the generalized eigenspace $E_{s_{0}}$ of the eigenvalue 0 of $D_{x} f\left(0, s_{0}\right)$ having dimension $l$ is perturbed to an invariant space $E_{s}$ of $D_{x} f(0, s)$ of dimension $l$ too, and all perturbed eigenvalues near 0 (the so-called 0-group) are eigenvalues of the finitedimensional operator $D_{x} f(0, s)$ restricted to the $l$-dimensional invariant space $E_{s}$. Moreover the eigenvalues in that 0 -group depend continuously on $s$. Motivated on these facts we can give the definition of odd crossing number.

Definition 5.1. Let $\Theta(s)=1$ if there are no negative real eigenvalues in the 0 -group of $D_{x} G(0, s)$, and

$$
\Theta(s)=(-1)^{l_{1}+\cdot+l_{h}}
$$

if $\mu_{1}, \ldots, \mu_{h}$ are all the negative real eigenvalues of the 0-group having algebraic multiplicity $l_{1}, \ldots, l_{h}$, respectively. If $D_{x} f(0, s)$ is regular in a neighborhood of $s_{0}$ (naturally except in the point $\left.s_{0}\right)$ and $\Theta(s)$ changes the sign at $s_{0}$ then $D_{x} f(0, s)$ has an odd crossing number at $s_{0}$.

In presence of an odd crossing number there exists a standard result known as the Krasnosel'skii Bifurcation Theorem (see [8] for the proof): 
Theorem 5.2. If $D_{x} f(0, s)$ has an odd crossing number at $s_{0}$, then $\left(0, s_{0}\right)$ is a bifurcation point for $f(x, s)=0$ with respect to the curve $\left\{(0, s) \mid s\right.$ in a neighborhood of $\left.s_{0}\right\}$.

Thank to this result, our main theorem 1.2 follows from the following proposition:

Proposition 5.3. $D_{v \|} G(0, T)$ has an odd crossing number at $T_{*}$.

Proof : We observe that we can write

$$
v^{\|}=\sum_{i=1}^{l} a_{k_{i}} \cos \left(k_{i} t\right)
$$

where $1=k_{1}<\cdots<k_{l}$. It is clear, from the definition of $G$, that $D_{v \|} G(0, T)$ preserves the eigenspaces, and

$$
D_{v \|} G(0, T)=\left.H_{T}\right|_{V_{k_{1}} \oplus \cdots \oplus V_{k_{l}}}
$$

Then the 0-group of eigenvalues is given by $\sigma_{k_{1}}(T), \ldots, \sigma_{k_{l}}(T)$, where $\sigma_{k_{1}}(T)=\sigma(T)$. For $T=T_{*}$ they are all equal to 0 . Moreover, by the proposition 3.2 only $\sigma_{k_{1}}(T)$ changes sign at $T_{*}$, and its associated eigenspace has dimension 1 . This means that $D_{v \|} G(0, T)$ has a crossing number at $T_{*}$ and completes the proof of the proposition.

\section{REFERENCES}

[1] H. Berestycki, L. A. Caffarelli and L. Nirenberg. Monotonicity for Elliptic Equations in Unbounded Lipschitz Domains, Communications on Pure and Applied Mathematics, Vol. L (1997), 1089-1111.

[2] F. Bowman. Introduction to Bessel Functions, Dover Publications Inc., New York, 1958.

[3] A. El Soufi and S. Ilias, Domain deformations and eigenvalues of the Dirichlet Laplacian in Riemannian manifolds, Illinois Journal of Mathematics 51 (2007) 645-666.

[4] P. R. Garadedian and M. Schiffer. Variational problems in the theory of elliptic partial differetial equations, Journal of Rational Mechanics and Analysis 2 (1953), 137-171.

[5] D. Gilbarg and N. S. Trudinger. Elliptic Partial Differential Equations of Second Order, Grundlehren der mathematischen Wissenschaften, a Series of Comprehensive Studies in Mathematics, Vol. 224, $2^{\text {nd }}$ Edition, Springer 1977, 1983.

[6] A. Gray and G.B. Mathews. A Treatise on Bessel Functions and Their Applications to Physics, Second Edition prepared by A. Gray and T.M. Macrobert, Dover Publications Inc., New York, 1966.

[7] T. Kato. Perturbation Theory for Linear Operator, Springer-Verlag, Berlin-Heidelberg-New York, 1987.

[8] H. Kielhofer. Bifurcation Theory, An Introduction with Applications to PDEs, Applied Mathematical Sciences, Vol. 156, Springer-Verlag, 2004.

[9] F. Pacard and P. Sicbaldi. Extremal domains for the first eigenvalue of the Laplace-Beltrami operator, Annales de l'Institut Fourier, Vol. $58 \mathrm{n}^{\circ} 2$ (2009).

[10] J. Serrin. A Symmetry Theorem in Potential Theory, Arch. Rational Mech. Anal. 43 (1971), 304-318.

[11] J. Smoller. Shock Waves and Reaction-Diffusion Equations, Grundlehren der mathematischen Wissenschaften, a Series of Comprehensive Studies in Mathematics, Vol. 258, $2^{\text {nd }}$ Edition, Springer 1983, 1994.

E-mail address: pieralberto.sicbaldi@univ-paris12.fr

Université Paris-Est, France 